\title{
Plasmon mechanism of the trion emission band broadening in quantum wells
}

\author{
A. A. Klochikhin, ${ }^{1,2, *}$ V. P. Kochereshko, ${ }^{1}$ L. Besombes,${ }^{3}$ G. Karczewski, ${ }^{4}$ T. Wojtowicz, ${ }^{4}$ and J. Kossut ${ }^{4}$ \\ ${ }^{1}$ Ioffe Physico-Technical Institute, 194021 St. Petersburg, Russia \\ ${ }^{2}$ Nuclear Physics Institute, 350000 St. Petersburg, Russia \\ ${ }^{3}$ Institut Néel, CNRS-Université Joseph Fourier, 38042 Grenoble, France \\ ${ }^{4}$ Institute of Physics, Polish Academy of Sciences, 02608 Warsaw, Poland
}

(Received 29 August 2010; revised manuscript received 18 April 2011; published 16 June 2011)

\begin{abstract}
The effects of charge-density fluctuations on negative trion states in a quantum well are considered for the case of intermediate doping by electrons at zero magnetic field. The quasi-two-dimensionality of the system and the trion-plasmon interaction are taken into account in the framework of the random-phase approximation. It is shown that the trion luminescence band has a complex structure consisting of a zero-plasmon line and a trion-plasmon wing. The structure is interpreted as a result of spatial inhomogeneity of the quantum wells.
\end{abstract}

DOI: 10.1103/PhysRevB.83.235329

PACS number(s): 71.10.Ca, 71.45.Gm, 73.21.Fg, 78.55.Et

\section{INTRODUCTION}

Trions, or negatively charged excitons, play an important role in determining the emission spectra of quantum wells (QW's) doped with electrons. A great number of spectroscopic studies of trions have been reported. ${ }^{1-11}$ However, until now there has been no explanation of the form of the trion photoluminescence (PL) spectrum in the situation in which the exciton binding energy dominates the Fermi energy of the background electron gas $E_{F}$ while the trion binding energy remains less than $E_{F}$. In particular, the shape of the trion luminescence band and the microscopic mechanism of its broadening, which is often larger than the trion's binding energy in the QW, have not been studied.

Experimental investigations of the trion band shape were performed for luminescence spectra observed in $\mathrm{CdTe} / \mathrm{CdMgTe}$ quantum wells at low temperatures. ${ }^{2,3,6,8-10}$ The relatively large trion and exciton binding energies in these wells enable us to investigate the trion's spectral characteristics in a wide interval of free-carrier concentration.

The photoluminescence spectrum of the trion in $\mathrm{CdTe} / \mathrm{CdMgTe}$ quantum wells at low electron density is represented by a relatively narrow inhomogeneously broadened line. The Stokes shift with respect to the line observed in the absorption spectrum is on the order of $1 \mathrm{meV}$. An increase in the electron concentration leads to the transformation of both photoluminescence and absorption spectra. We consider the PL spectrum, which is represented in this case by a wide band. The characteristic feature of this band is the clearly visible trace of the sharp peak at the high-energy side of the band, the energy position of which does not depend on the electron concentration and coincides with the trion's line in the limit of low electron density. The latter circumstance allows us to consider the PL band as a result of the trion emission rather than the band-to-band recombination with a Fermi edge singularity. An effect of charged trion formation is polarization of free electrons. This can be described as an infinite succession of the scattering processes of the trion from electron-hole pairs due to Coulomb interaction. We consider this effect in the random-phase approximation. As a result, we obtain the trion state affected by the polarized environment.

The microscopic mechanisms responsible for the creation of the pair excitation can be the Coulomb or exchange interaction of the trion with free carriers. For the charged trion, the Coulomb interaction seems to be the only mechanism because in the absence of magnetic fields, the triplet trion is unbound over a wide range of electron and hole mass ratio. ${ }^{10,12}$

The free trion that emits a photon has an almost zero wave vector. Scattering of the trion by free electrons, with excitation of an electron-hole pair in the conduction band near the Fermi level at $p \approx p_{F}$ with the wave vector of its center-of-mass motion $\mathbf{q}$, creates a pair with energy $\left(\hbar^{2} q^{2} / 2 m+\hbar^{2} \mathbf{p q} / m\right)$. The threshold energy of this excitation at $q=0$ is zero. Therefore, the boundary of the continuum spectrum of the combined excitation consisting of the free trion plus the pair excitation coincides with the energy of the photon emitted by the free trion. As a consequence of this inelastic-scattering process, the trion self-energy will be renormalized and the trion luminescence (and absorption) band will be homogeneously broadened.

It is well known that in a wide interval of wave vectors, the electron gas excitations are strongly renormalized due to the electron-electron Coulomb interactions, so that the plasmon begins to play a leading role in the excitation spectrum. ${ }^{13-18}$ Investigations of quasi-two-dimensional plasmon excitations and their role in the energy-loss processes were performed in Refs. 18-20. We will show that such energy-loss processes are responsible for the trion self-energy correction and the emission band broadening.

We consider the behavior of the trion luminescence band as a function of free-electron concentration in $\mathrm{CdTe} / \mathrm{CdMgTe}$ quantum wells where the Fermi energy is small in comparison with the binding energy of the quasi-two-dimensional exciton. The electron concentration dependence of the broadening allows one to relate the process to the interaction of the trion with the wave-vector-dependent plasmon, which is a fundamental elementary excitation and a collective density oscillation mode.

\section{THEORY}

We consider the quantum well as a quasi-two-dimensional system and transform the Coulomb interaction to take account of the lowered dimensionality due to confinement. Some minor simplifications are useful to minimize the size of the calculation. 
First, we assume that all of the particles (electrons and trions) occupy their lowest confined states in a quantum well with infinite barriers. In this case, the wave functions of all of the particles can be characterized by the single length $L$, which is defined by the quantum-well thickness. A further simplification is related to the description of the trion's internal motion: The trion is considered as a compound particle consisting of an exciton and a weakly bound electron. We consider only low temperatures with $k_{B} T$ much less than the Fermi energy. This makes analytical calculations possible up to the last integral, which is calculated numerically.

In the case of the trion confined in a quantum well, the wavevector conservation law exists only for the in-plane center-ofmass motion of this particle. The free electrons interacting with the trion are also confined in the quantum well and, therefore, only the in-plane components of their wave vectors obey the conservation law.

\section{A. Coulomb interaction in a quantum well}

It is convenient to deduce the necessary equations using as a starting point the Coulomb interaction in a three-dimensional (3D) system,

$H_{\text {Coul }}=\frac{1}{2} \sum_{i, j, \sigma, \sigma^{\prime}} \int d^{3} r_{i} d^{3} r_{j} V_{\text {Coul }}\left(\mathbf{r}_{i}-\mathbf{r}_{j}\right)\left|\psi_{\sigma}\left(\mathbf{r}_{i}\right)\right|^{2}\left|\psi_{\sigma^{\prime}}\left(\mathbf{r}_{j}\right)\right|^{2}$.

Here, $\sigma$ denotes the spin quantum number and

$$
V_{\text {Coul }}\left(\mathbf{r}_{1}-\mathbf{r}_{2}\right)=\frac{1}{(2 \pi)^{3}} \int d^{3} q \frac{4 \pi e^{2}}{\varepsilon q^{2}} \exp \left[i \mathbf{q}\left(\mathbf{r}_{1}-\mathbf{r}_{2}\right)\right]
$$

where $\varepsilon$ is the static dielectric constant, and the $\mathbf{r}_{i}$ with $i=1,2$ are the radius vectors of two interacting particles. Writing Eq. (2) and below, we have used CGS units.

In the case of quasi-two-dimensional quantum wells, it is convenient to introduce the in-plane and perpendicularto-plane coordinates $\mathbf{r} \rightarrow(\vec{\rho}, z)$ and the corresponding wave vectors $\mathbf{q} \rightarrow\left(\hat{\mathbf{q}}, q_{z}\right)$ in the reciprocal space.

The simplified trion wave function is assumed to have the form of the product of the wave function for the free motion of the center of mass $\Psi(\vec{R}) \sim \exp (i \hat{\mathbf{q}} \vec{R})$, where $\vec{R}$ is the two-dimensional center-of-mass coordinate, and the wave function of the electron bound to the exciton $\psi_{\mathrm{tr}} \sim$ $\exp \left(-|\vec{R}-\vec{\rho}| / a_{\text {tr }}\right)$, where $a_{\text {tr }}$ is a radius characterizing the electron motion in the trion.

The integral over $d^{2} \mathcal{R}$ in the scattering amplitude produces the wave-vector conservation law for the in-plane components of the center-of-mass wave vector. As result, the two-dimensional wave vector of the trion's center of mass is equal to the in-plane wave-vector component of the Coulomb vertex.

The integral over $d^{2} \rho$ of the product of the square modulus of the wave functions describing the trion's internal motion and the exponential function is transformed to

$$
\begin{aligned}
\mathcal{M}_{\mathrm{tr}}(\hat{q}) & =\int d^{2} \rho\left|\psi_{\mathrm{tr}}(\rho)\right|^{2} \exp (i \hat{\mathbf{q}} \vec{\rho}) \\
& =\left\{\frac{2+\left(\hat{q} a_{\mathrm{tr}} / 2\right)^{2}}{2\left[1+\left(\hat{q} a_{\mathrm{tr}} / 2\right)^{2}\right]^{3 / 2}}\right\} .
\end{aligned}
$$

This form factor arises twice when the Coulomb interaction of the electron-hole pair with the trion is included.

We exclude the motion in the perpendicular direction by calculating the matrix elements of exponentials with wave functions $\psi(z)=\sqrt{2 / L} \cos (\pi z / L)$, assuming that the wave functions of the trion and electron-hole pairs are completely confined within the quantum well of thickness $L$ restricted by infinite barriers. Both matrix elements have the form

$$
M_{z, z}=\int_{-L / 2}^{L / 2} d z|\psi(z)|^{2} \exp \left(i z q_{z}\right)=\frac{2}{q_{z} L} \sin \left(q_{z} L / 2\right),
$$

which can be written as an infinite product

$$
M_{z, z}\left(q_{z}\right)=\prod_{k=2}^{\infty}\left[1-\left(q_{z} L\right)^{2} /(2 \pi k)^{2}\right] .
$$

The dependence of $M_{z, z}\left(q_{z}\right)$ on $q_{z}$ is presented by curve 1 in Fig. 1.

Finally, the quasi-2D matrix element of the Coulomb interaction can be found by calculating the integral over $d q_{z}$,

$$
V(\hat{q})^{2 \mathrm{D}}=\int_{0}^{\infty} \frac{d q_{z}}{2 \pi} \frac{4 \pi e^{2}\left[M_{z, z}\left(q_{z}\right)\right]^{2}}{\varepsilon\left[\hat{q}^{2}+q_{z}^{2}\right]},
$$

which can be calculated numerically (curve 2 in Fig. 1) or represented with good accuracy by the function (curve 3 in Fig. 1)

$$
V(\hat{q})^{2 \mathrm{D}}=\frac{4 e^{2}}{\varepsilon \hat{q}} \arctan \frac{\widetilde{q}_{L}}{\hat{q}},
$$

where $\widetilde{q}_{L}=2 \pi / \widetilde{L}$ and

$$
\frac{L}{\widetilde{L}}=\int_{0}^{\infty} \frac{L d q_{z}}{2 \pi}\left[M_{z, z}\left(L q_{z} / 2 \pi\right)\right]^{2} \simeq 0.75
$$

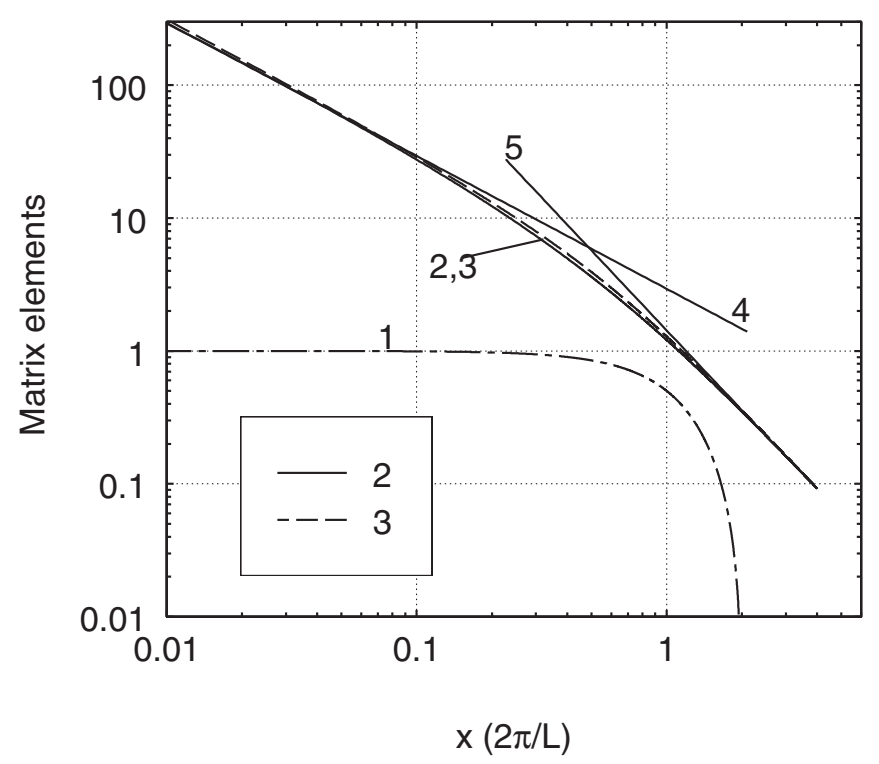

FIG. 1. Curve 1 is the matrix element $M_{z, z}(x)=\prod_{k=2}^{N}[1-$ $\left.x^{2} / k^{2}\right]$ at $N=100$, solid curve 2 is the result of numerical calculation of $\int_{0}^{\infty} d x^{\prime} \frac{\left|M_{z, z}^{\mathrm{tr}}\left(x^{\prime}\right)\right|^{2}}{\left[x^{\prime 2}+x^{2}\right]}$, and dashed curve 3 is an approximation of curve 2 by the function $\left[\frac{2}{x} \arctan \frac{L}{\tilde{L} x}\right]$. Curves 4 and 5 are asymptotes $\pi / x$ and $2 L / \widetilde{L} x^{2}$. 
The value $\widetilde{L} \simeq 4 L / 3$ represents the distance where the character of the Coulomb interaction changes.

In the limit of small $\hat{q}$, we obtain the two-dimensional Coulomb interaction from Eq. (7) (see, for example, Chap. 9 of Ref. 13) as

$$
\left.V(q)^{2 \mathrm{D}}\right|_{\hat{q} \rightarrow 0}=\frac{2 \pi e^{2}}{\varepsilon \hat{q}} .
$$

In the limit of large $\hat{q}$, Eq. (7) transforms into the 3D Coulomb matrix element

$$
\left.V(q)^{2 \mathrm{D}}\right|_{\hat{q} \gg \widetilde{q}_{L}}=\frac{4 \pi e^{2}}{\varepsilon \hat{q}^{2}(\widetilde{L} / 2)}
$$

(see asymptotes in Fig. 1). Therefore, $\widetilde{L} / 2$ is the effective thickness of the quasi-two-dimensional slab for this model.

\section{B. The polarization loop and the dielectric function}

The polarization loop of the electron-electron Coulomb interaction for degenerate 2D electrons in the low-temperature limit (see Fig. 2 and Table I) is expressed as

$$
P_{\hat{q}}^{\text {Coul }}(\hbar \omega)=-V^{2 \mathrm{D}}(\hat{q}) \chi(\hat{q}, \hbar \omega) .
$$

Here, the complex function $\chi(\hat{q}, \hbar \omega)$ is

$$
\begin{aligned}
\chi(\hat{q}, \hbar \omega)= & -\int_{0}^{p_{F}} p d p \int_{0}^{2 \pi} \frac{d \varphi}{2 \pi} \\
& \times\left\{\frac{1}{\hbar \omega+i \delta-\omega_{\hat{\mathbf{p}} \hat{\mathbf{q}}}}-\frac{1}{\hbar \omega+i \delta+\omega_{\hat{\mathbf{p}} \hat{\mathbf{q}}}}\right\},
\end{aligned}
$$

where $\omega_{\hat{\mathbf{p}} \hat{\mathbf{q}}}=\hbar^{2}(\hat{\mathbf{p}} \hat{\mathbf{q}}) / m^{*}+\hbar^{2} \hat{q}^{2} / 2 m^{*}, m^{*}$ is the electron effective mass, $\delta \rightarrow 0, p_{F}$ is the Fermi wave vector, and $\varphi$ is the angle between two-dimensional vectors $\hat{\mathbf{p}}$ and $\hat{\mathbf{q}}$.

In the limit $\omega \rightarrow 0$, the result coincides with the ThomasFermi approximation

$$
\operatorname{Re} \chi(\hat{q}, 0)=-\frac{m^{*}}{\pi \hbar^{2}} .
$$

In the limit $\hat{q} \ll q_{L}$, we obtain for $\operatorname{Re} P_{\hat{q}}^{\text {Coul }}(0)$ the well-known expression $^{13}$

$$
\operatorname{Re} P_{\hat{q}}^{\text {Coul }}(0)=\frac{2}{a_{B} \hat{q}},
$$

where $a_{B}$ is the Bohr radius of the $3 \mathrm{D}$ exciton.

It is convenient to introduce the universal dimensionless susceptibility function $\tilde{\chi}\left(\hat{q} / p_{F},(\hbar \omega) / E_{F}\right)$

$$
\chi(\hat{q}, \hbar \omega)=\frac{p_{F}^{2}}{2 \pi E_{F}} \tilde{\chi}\left(\hat{q} / p_{F},(\hbar \omega) / E_{F}\right) .
$$

Calculating the integral

$$
-\frac{1}{2 \pi E_{F}^{2}} \int_{0}^{\infty} \operatorname{Im} \chi(\hat{q}, \hbar \omega) \hbar \omega d \hbar \omega=\hat{q}^{2} / p_{F}^{2},
$$

where $E_{F}$ is the Fermi energy, we obtain one more relation that can be used together with Eqs. (12) and (13) to check the calculations of $\chi(\hat{q}, \hbar \omega)$.

In our calculations, the function $\operatorname{Im} \chi(\hat{q}, \hbar \omega)$ was obtained analytically similarly to calculations of the dielectric function in Ref. 14, while $\operatorname{Re} \chi(\hat{q}, \hbar \omega)$ was found as the result of the Hilbert transformation.
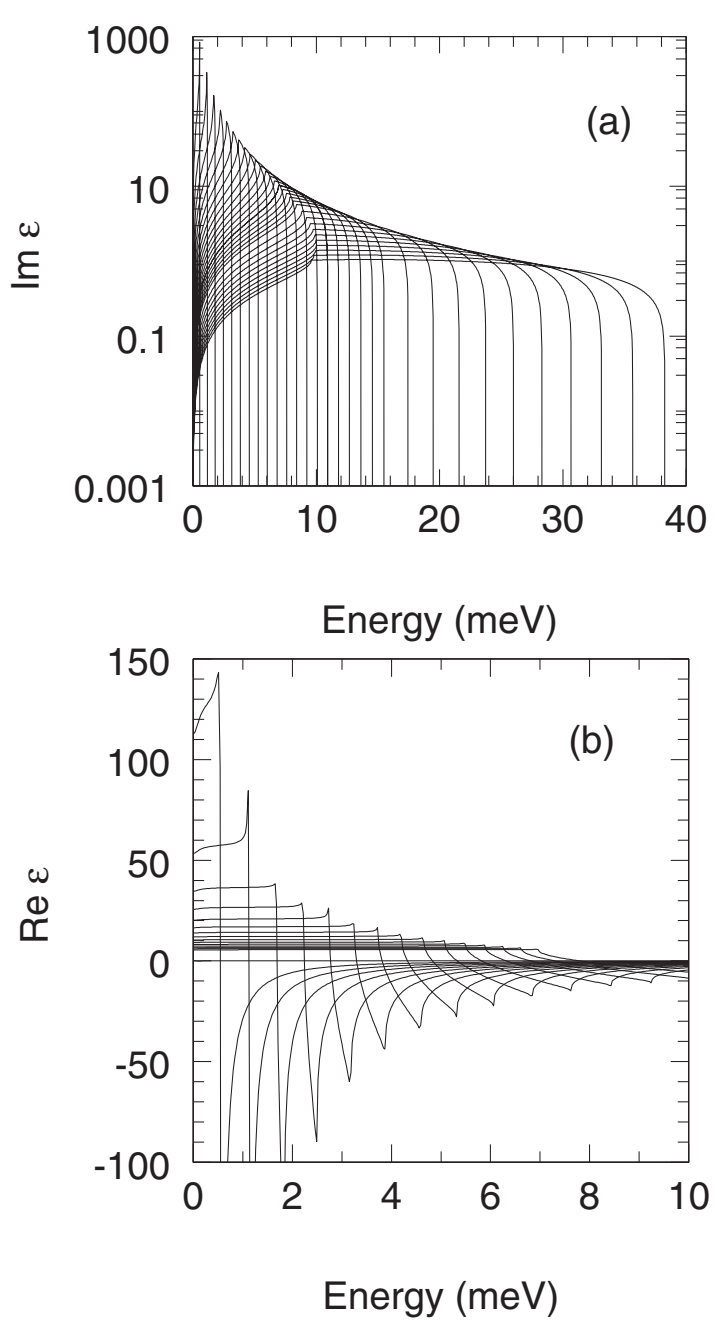

FIG. 2. Imaginary part (a) and real part (b) of the 2D polarizability $P_{\hat{q}}^{\mathrm{Coul}}(\hbar \omega)$ for $E_{F}=10 \mathrm{meV}$. The values $\hat{q}$ are taken in the interval from 0 to $0.75 p_{F}$. The calculations use $\mathrm{CdTe} / \mathrm{CdMgTe}$ quantum-well parameters.

\section{The trion self-energy correction}

The trion self-energy correction due to the Coulomb interaction with electrons is expressed through the integral over $\hat{q}$ of the polarization loop multiplied by the matrix element of the Coulomb interaction. This integral is divergent at small $\hat{q}$ even in the quasi-two-dimensional case. The problem to be solved is the renormalization of the polarization loop by the electron-electron Coulomb interaction. In other words, we have to include the dynamical screening of the Coulomb vertex to remove the divergence of the first-order self-energy correction at small wave vectors. This can be done in the random-phase approximation. As a result, the self-energy

TABLE I. Parameters used in the calculations: the electron mass, the CdTe 3D exciton binding energy $E_{\mathrm{ex}}^{3 \mathrm{D}}$, the trion binding energy $E_{\text {tr }}^{B}$, and the effective trion radius.

\begin{tabular}{lcccc}
\hline \hline$m_{e}$ & $E_{\mathrm{ex}}^{3 \mathrm{D}}(\mathrm{meV})$ & $E_{\mathrm{tr}}^{B}(\mathrm{meV})$ & $a_{B}^{3 \mathrm{D}}(\AA)$ & $a_{\mathrm{tr}}(\AA)$ \\
\hline $0.11 m_{0}$ & 10 & 2 & 70 & 200 \\
\hline \hline
\end{tabular}


correction to the trion energy at zero wave vector $E_{\mathrm{tr}}(0)$ can be represented as

$\delta E_{\mathrm{tr}}(\hbar \omega)=\frac{1}{(2 \pi)^{2}} \int d^{2} q \frac{V^{2 D}(\hat{q})\left[\mathcal{M}_{\mathrm{tr}}(\hat{q})\right]^{2}}{1-V^{2 D}(\hat{q}) \chi\left(\hat{q}, \hbar \omega-\hbar^{2} \hat{q}^{2} / 2 M_{\mathrm{tr}}\right)}$,

where $\hbar^{2} \hat{q}^{2} / 2 M_{\mathrm{tr}}=E_{\mathrm{tr}}(\hat{q})-E_{\mathrm{tr}}(0)$ and $M_{\mathrm{tr}}$ is the trion effective mass. The energy of the trion at zero wave vector is taken as a frame of reference for $\hbar \omega$. The imaginary part of Eq. (16) representing damping of the trion state is

$$
\begin{aligned}
\Gamma(\omega)= & \operatorname{Im}\left[\delta E_{\mathrm{tr}}(\hbar \omega)\right] \\
= & \frac{1}{(2 \pi)^{2}} \int d^{2} q \frac{4 e^{2} \arctan \frac{\widetilde{q}_{L}}{\hat{q}}\left[\mathcal{M}_{\mathrm{tr}}(\hat{q})\right]^{2}}{\hat{q}} \\
& \times \operatorname{Im}\left[\frac{1}{\varepsilon\left(\hat{q}, \hbar \omega-\hbar^{2} \hat{q}^{2} / 2 M_{\mathrm{tr}}\right)}\right],
\end{aligned}
$$

where

$$
\begin{aligned}
& \varepsilon\left(\hat{q}, \hbar \omega-\hbar^{2} \hat{q}^{2} / 2 M_{\mathrm{tr}}\right) \\
& \quad=\varepsilon\left[1-\frac{4 e^{2}}{\varepsilon \hat{q}} \arctan \frac{\widetilde{q}_{L}}{\hat{q}} \chi\left(\hat{q}, \hbar \omega-\hbar^{2} \hat{q}^{2} / 2 M_{\mathrm{tr}}\right)\right] .
\end{aligned}
$$

Equation (17) can be calculated directly in the continuum spectrum region where $\operatorname{Im} \chi\left(\hat{q}, \hbar \omega-\hbar^{2} \hat{q}^{2} / 2 M_{\mathrm{tr}}\right) \neq 0$. Special consideration should be given to inclusion of the plasmon pole contribution. The energies of the plasmon poles $\hbar \omega_{\mathrm{pl}}(\hat{q})$ are defined by zeros of the function $\left[1-V^{2 \mathrm{D}}(\hat{q}) \chi(\hat{q}, \hbar \omega)\right]$ in the region where $\operatorname{Im} \chi\left(\hat{q}, \hbar \omega-\hbar^{2} \hat{q}^{2} / 2 M_{\text {tr }}\right)=0$. The plasmon contribution to the reciprocal dielectric function dominates in the whole region of existence of the plasmon, as can be seen in Fig. 3(a).

The weight of the plasmon contribution to the reciprocal dielectric function is defined by the residue of the real part of

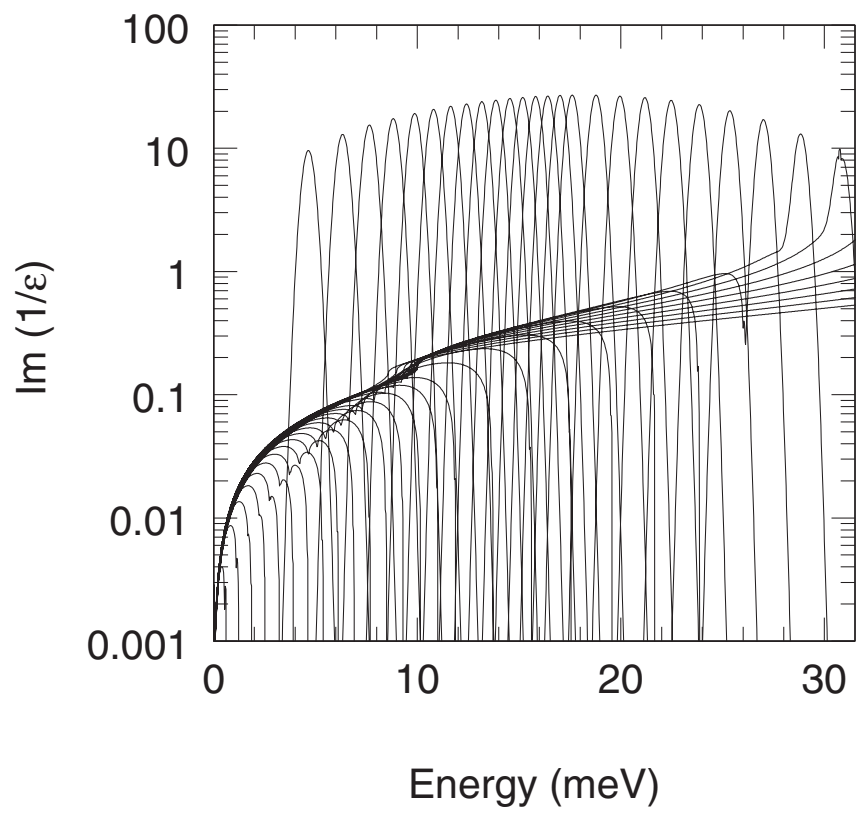

FIG. 3. The imaginary part of the reciprocal dielectric function $1 / \varepsilon(\hat{q}, \hbar \omega)$ in the plasmon region, for $E_{F}=10 \mathrm{meV}$.

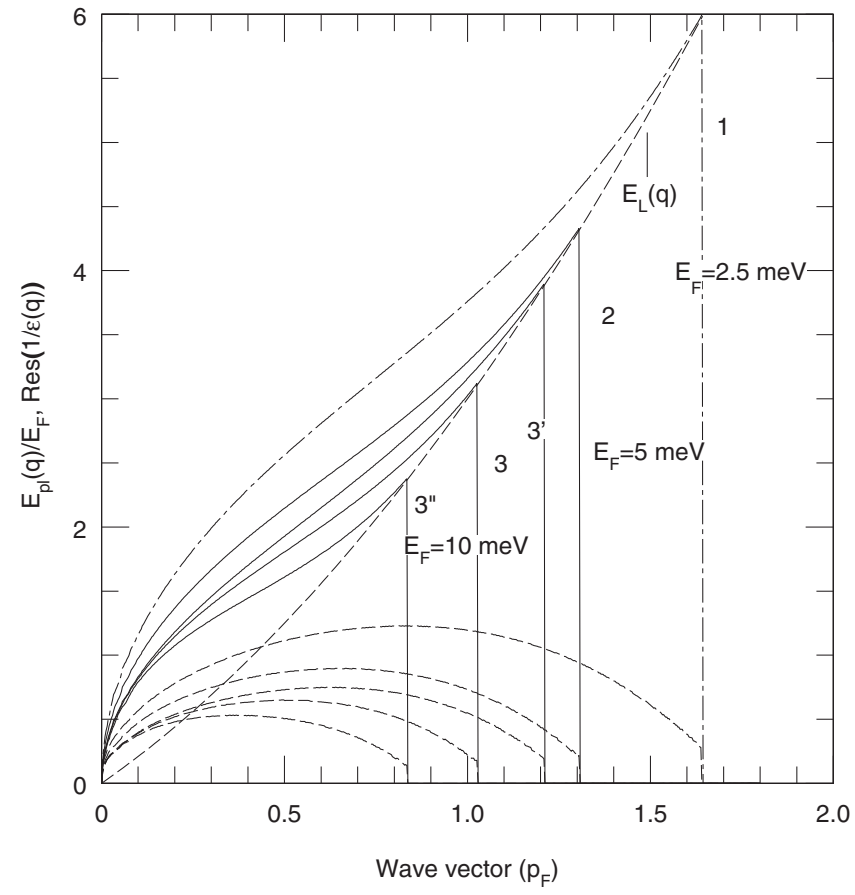

FIG. 4. Dispersion of the plasmon excitation branch $\hbar \omega_{\mathrm{pl}}(q)$ for values of the Fermi energy 2.5, 5, and $10 \mathrm{meV}$ (curves from 1 to 3 , respectively). Dashed curve $E_{L}$ is the Landau damping boundary. Residues $\operatorname{Res}_{\omega}(1 / \varepsilon(\hat{q}, \hbar \omega))$ of the plasmon branch for corresponding Fermi energies are represented by dashed curves. Curves 1-3 are calculated for quantum-well thickness equal to $10 \mathrm{~nm}$, while curves $3^{\prime}$ and $3^{\prime \prime}$ are for thicknesses 5 and $20 \mathrm{~nm}$, respectively. The calculations use $\mathrm{CdTe} / \mathrm{CdMgTe}$ quantum-well parameters.

the dielectric function,

$$
\operatorname{Res}_{\omega}(1 / \varepsilon(\hat{q}, \hbar \omega))=\left[\frac{d}{d \omega} \operatorname{Re}(\varepsilon(\hat{q}, \hbar \omega))\right]_{\omega=\omega_{\mathrm{pl}}(\hat{q})}^{-1},
$$

calculated at $\omega=\omega_{\mathrm{pl}}(\hat{q})$. Functions $\omega_{\mathrm{pl}}(\hat{q})$ and $\operatorname{Res}_{\omega}(1 /$ $\varepsilon(\hat{q}, \hbar \omega))$ are presented in Fig. 4 .

Taking the trion energy at wave vector $k=0$ as a point of reference for energy, we represent the plasmon contribution to the imaginary part of the trion's self-energy correction at photon energy $\hbar \omega$ as

$$
\begin{aligned}
\operatorname{Im}\left[\delta E_{\mathrm{tr}}(\hbar \omega)\right]= & \int \frac{d^{2} \hat{q}}{(2 \pi)^{2}}\left\{\frac{4 e^{2} \arctan \frac{\widetilde{q}_{L}}{\hat{q}}\left[\mathcal{M}_{\mathrm{tr}}(\hat{q})\right]^{2}}{\hat{q}\left[\frac{d}{d \omega} \operatorname{Re}(\varepsilon(\hat{q}, \omega))\right]_{\omega=\omega_{\mathrm{pl}}(\hat{q})}}\right\} \\
& \times \pi \delta\left(\omega-\hbar \hat{q}^{2} / 2 M_{\mathrm{tr}}-\omega_{\mathrm{pl}}(\hat{q})\right) .
\end{aligned}
$$

The plasmon broadening remains undefined in the randomphase approximation, and serious care should be taken to avoid the influence of artificial plasmon broadening of the properties of the reciprocal dielectric function. The most appropriate check can be realized by the sum rule

$$
\frac{1}{2 \pi} \int d \omega \operatorname{Im}[\omega \varepsilon(\hat{q}, \omega)]=\frac{1}{2 \pi} \int d \omega \operatorname{Im}\left[\frac{\omega}{\varepsilon(\hat{q}, \omega)}\right],
$$

which reflects the electronic charge conservation and provides the gauge invariance of the approach. The plasmon contribution of Eq. (20) to the trion's self-energy correction should 
be included together with the contribution of the continuum spectrum of Eq. (17).

In the calculations, the shape of the plasmon line should be approximated by a function that makes overlapping of the plasmon line and continuum negligible. The normalized Gaussian function is appropriate for this purpose. In Fig. 3, the broadening of the Gaussians is taken to be independent of $\hat{q}$ and large enough to make the overlapping of discrete and continuum spectra visible at large wave vectors where the splitting of the plasmon poles decreases.

The plasmon dispersion curves at different Fermi energies and different quantum-well thicknesses are presented in Fig. 4. The region of existence of the plasmon is restricted by the Landau damping boundary (curve $E_{L}$ in Fig. 4). It is seen that the region is not universal and depends on the product $\left(p_{F} L\right)$. In terms of $p_{F}$, it decreases as the product $\left(p_{F} L\right)$ increases. The plasmon dispersion at small wave vectors can be approximated by a square-root dependence. In the calculations, we have neglected the trion kinetic energy (recoil effect), which is small compared to the plasmon energy.

The real part of Eq. (17) calculated by using the Hilbert transformation gives the dynamical shift of the trion state due to electron-electron Coulomb interaction,

$$
\begin{aligned}
\Delta(\omega)= & \operatorname{Re}\left[\delta E_{\mathrm{tr}}(\hbar \omega)\right] \\
= & \frac{1}{(2 \pi)^{2}} \int d^{2} q \frac{4 e^{2} \arctan \frac{\widetilde{q}_{L}}{\hat{q}}\left[\mathcal{M}_{\mathrm{tr}}(\hat{q})\right]^{2}}{\hat{q}} \\
& \times\left\{\operatorname{Re}\left[\frac{1}{\varepsilon\left(\hat{q}, \hbar \omega-\hbar^{2} \hat{q}^{2} / 2 M_{\mathrm{tr}}\right)}\right]\right\} .
\end{aligned}
$$

It is seen from Eqs. (16)-(19) that the trion self-energy correction is defined through the spectrum of the chargedensity fluctuations describing the energy losses of the trion.

The imaginary part of $1 / \varepsilon\left(\hat{q} / p_{F}, \hbar \omega / E_{F}\right)$ is presented in Fig. 3. As is seen, the spectrum of the energy losses consists of a continuum and a discrete spectrum formed by plasmons. The renormalization performed on the single polarization loop has resulted in the drastic decrease of the continuum contribution as compared with the polarization loop in Fig. 2(a).

\section{Trion band broadening (theory)}

We represent the shape of the trion luminescence line without interaction with charge-density fluctuations (the zeroplasmon line) via the spectral density

$$
I_{\mathrm{PL}}^{0}(\omega) \sim \frac{1}{\pi} \frac{\gamma_{0}}{\left(\hbar \omega-E_{\mathrm{tr}}\right)^{2}+\gamma_{0}^{2}},
$$

where $E_{\mathrm{tr}}$ is the trion energy at $\hat{q}=0, \gamma_{0}=1 / \tau, 1 / \tau=$ $\left(1 / \tau_{r}+1 / \tau_{\mathrm{qel}}\right), 1 / \tau$ is the full lifetime due to radiative and quasielastic processes, $\tau_{r}$ is the radiative lifetime, and $\tau_{\text {qel }}$ is the lifetime due to quasielastic processes. The factor $\tau_{r} / \tau$ defines the fraction of trions participating in the formation of the zero-plasmon PL band during the trion lifetime. The zero-plasmon line can be essentially suppressed by the energy relaxation of the trion state.

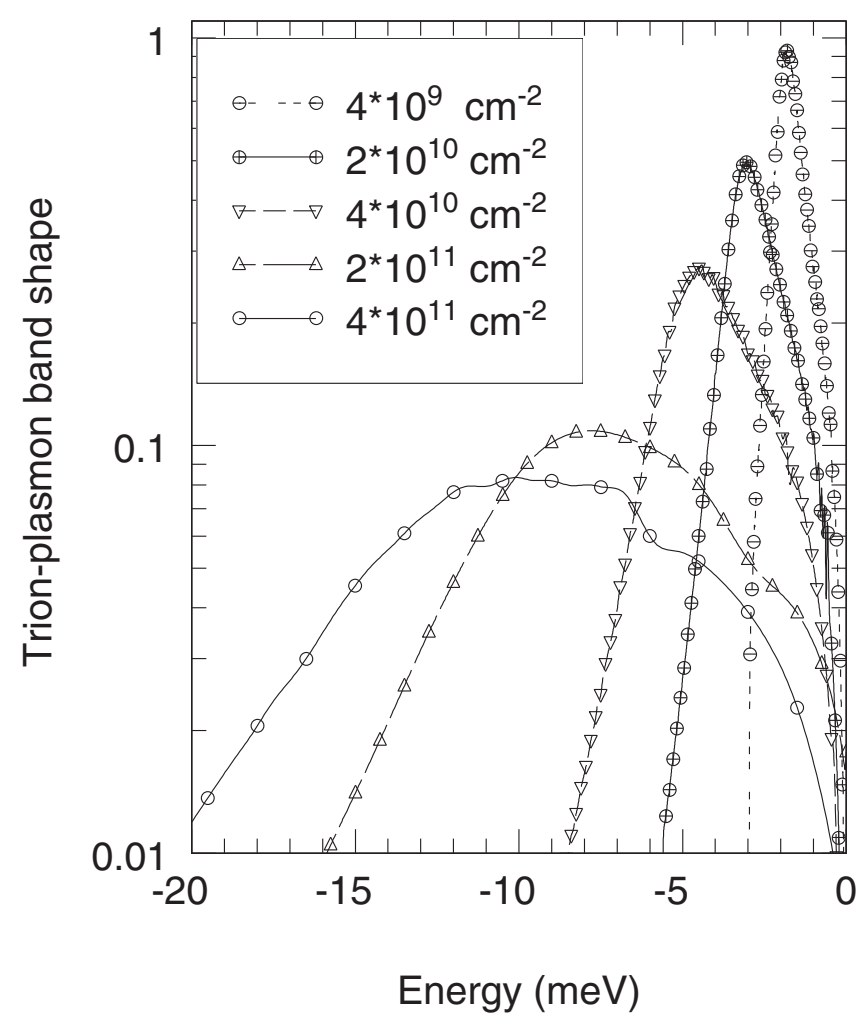

FIG. 5. The trion luminescence spectra calculated by using Eq. (24) for quantum wells with different electron densities corresponding to the Fermi energy variation from 0.1 to $10 \mathrm{meV}$. The $\mathrm{CdTe} / \mathrm{CdMgTe}$ quantum-well parameters given in Table I and the quantum-well thickness equal to $10 \mathrm{~nm}$ are used in the calculations. The zero of energy corresponds to the trion position without taking into account the trion-electron-hole interaction.

The shape of the trion band taking into account the interaction with charge-density fluctuations $(\mathrm{CDF})$ can be written as

$$
I_{\mathrm{PL}}^{\mathrm{CDF}} \sim \frac{1}{\pi} \frac{\gamma_{0}+\Gamma_{\mathrm{tr}}(\omega)}{\left[\hbar \omega-E_{\mathrm{tr}}-\Delta_{\mathrm{tr}}(\omega)\right]^{2}+\left[\gamma_{0}+\Gamma_{\mathrm{tr}}(\omega)\right]^{2}},
$$

where $\Gamma_{\mathrm{tr}}(\omega)$ is defined by the sum of Eqs. (17) and (20), and $\Delta_{\text {tr }}(\omega)$ is given by Eq. (22), respectively.

Results of calculations by using Eq. (24) for different freeelectron densities are presented in Fig. 5. As is seen in this figure, the trion spectrum forms a band whose width increases sharply with the increasing electron density. The maximum of the band shifts considerably to low energy.

We note that the contribution of the trion-plasmon interaction to the function $\Gamma_{\mathrm{tr}}(\omega)$ in the limit of $\omega \rightarrow 0$ goes to zero as $\omega^{2}$. The contribution of the interaction with the continuum electron-hole spectrum can be important in this limit. However, at increasing $\omega$ the trion-plasmon interaction becomes completely dominant.

The physical sense of $\Gamma_{\mathrm{tr}}(\omega)$ is the probability of inelastic scattering of the trion accompanied by the transition from the initial state with wave vector $\hat{k} \approx 0$ to the continuum set of final states, each of which is the combined excitation consisting of the trion with wave vector $\hat{k}+\hat{q} \approx \hat{q}$ and the plasmon with wave vector $-\hat{q}$. 
We consider this process to be the fastest one among the trion decay processes so that $\tau \Gamma_{\mathrm{tr}}(\omega) \gg 1$. The maximum value of $\Gamma_{\mathrm{tr}}(\omega)$ arises from the contribution of $\hat{q} \approx 1 / a_{\mathrm{tr}}$. As a rule, in the frequency region $\omega>\gamma_{0}$, the plasmon contribution dominates over the quasielastic and radiative processes.

\section{EXPERIMENTAL DATA AND DISCUSSION}

\section{A. Experiment}

We have used in this work two sets of $\mathrm{CdTe} / \mathrm{Cd}_{0.7} \mathrm{Mg}_{0.3} \mathrm{Te}$ heterostructures grown during two molecular-beam epitaxy processes on (100) GaAs substrates. Samples of two sets contain a single QW whose width was 100 or $80 \AA$, respectively. In the first set presented in Figs. 6 and 7, the QW width was $100 \AA$ A. In the second set presented in Fig. 8, the width was $80 \AA$. A series of these QW's was grown during a single epitaxial growth run using "wedge doping" techniques. ${ }^{21}$ To minimize dislocations due to a lattice mismatch between the QW and barrier materials, the QW was grown after a 400-nm-thick CdTe buffer layer.

An iodine $n$-type $\delta$ layer is located at a distance of $100 \AA$ from the QW. At low temperatures, electrons from the $\delta$ layer collect in the $\mathrm{QW}$, forming a quasi-2D electron gas.

All the QW's of the series have the same parameters except that they have different doping levels in the $\delta$ layer and, therefore, different electron gas densities. The preliminary estimation ${ }^{10}$ has shown that the electron concentrations in

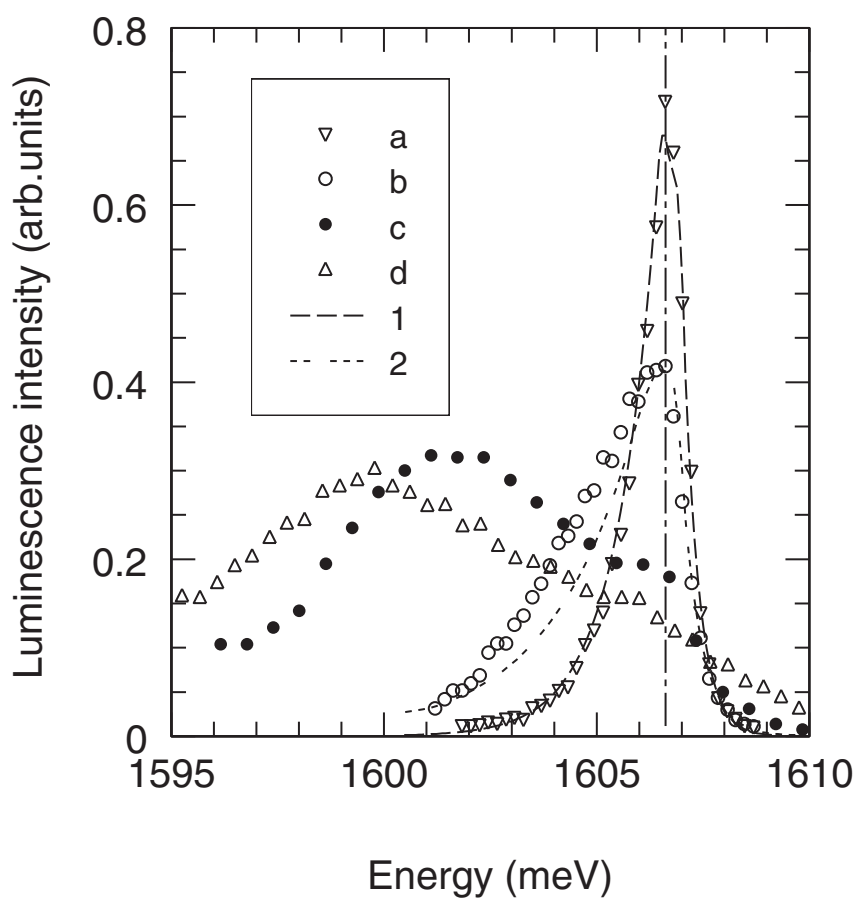

FIG. 6. The transformation of the trion luminescence spectrum taken from a CdTe/CdMgTe quantum well with increasing electron density (curves from $a$ to $d$ ). The quantum-well thickness is equal to $10 \mathrm{~nm}$. Symbols are experimental data, curves 1 and 2 are approximations of the inhomogeneous shapes by Eq. (23) using the distribution of $E_{\text {tr }}$ given by Eq. (25) with different values of $\gamma_{\text {inh }}$ above and below $E_{\mathrm{tr}}^{0}$. The vertical line represents the spectral position of the zero-plasmon trion line $E_{\mathrm{tr}}^{0}$ in the low-electron-density limit.

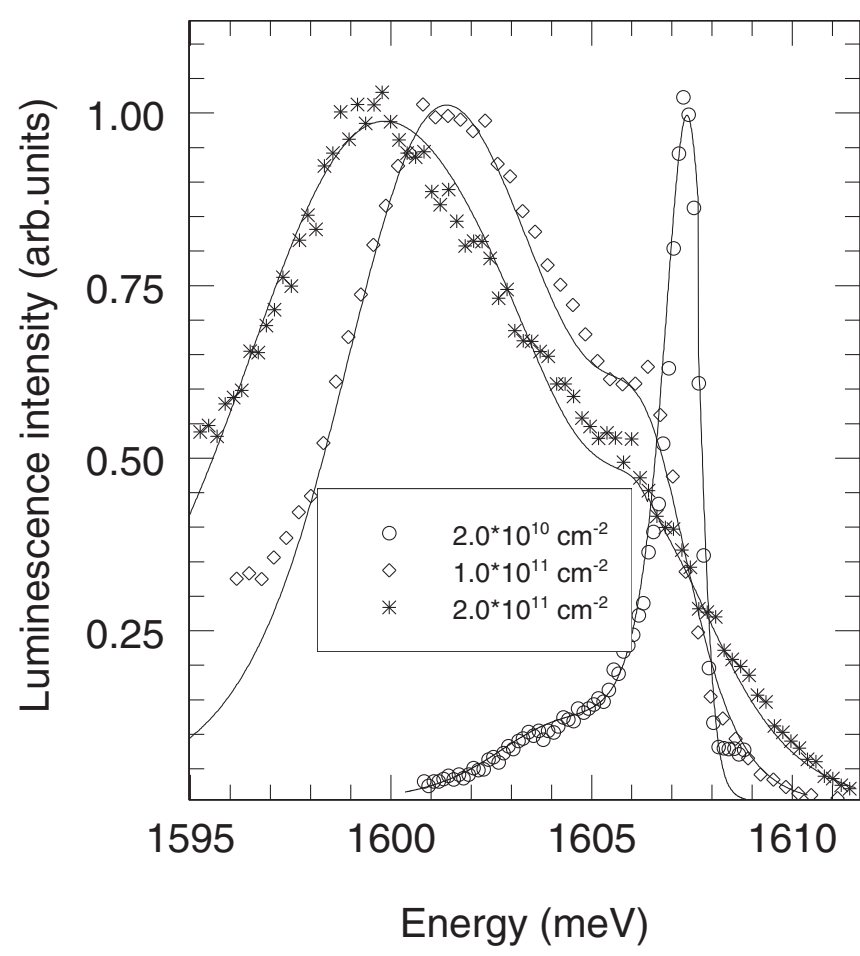

FIG. 7. Luminescence spectra taken from $\mathrm{CdTe} / \mathrm{CdMgTe}$ quantum wells with different electron concentrations. Symbols are the experimental data. Curves (solid lines) are approached by using linear combinations of the inhomogeneously broadened zero-plasmon band of Eq. (23) and by Eq. (24) for different electron densities. The trion spectral position $E_{\text {tr }}$ is averaged over the distribution Eq. (25) with different values of $\gamma_{\text {inh }}$ above and below $E_{\mathrm{tr}}^{0}$. The CdTe/CdMgTe quantum-well parameters given in Table I are used in calculations. The quantum-well thickness is equal to $10 \mathrm{~nm}$.

the QW's are in the interval between $10^{10}$ and $10^{12} \mathrm{~cm}^{-2}$. (We indicate here the concentration that follows from the level of the doping impurities; the real concentration could be smaller.) The samples were not photosensitive, i.e., the electron concentration did not depend on the intensity of additional illumination.

The PL spectra were excited by an Ar-ion laser with $\lambda=$ $530 \mathrm{~nm}$ and recorded by a $1 \mathrm{~m}$ spectrometer with a chargecoupled device (CCD) detector. The excitation power did not exceed $100 \mathrm{~W} / \mathrm{cm}^{2}$.

\section{B. Trion band broadening (experiment and theory)}

The luminescence spectra for a set of samples with different electron densities are presented in Fig. 6. As is seen, the spectra at low electron densities (curves $a$ and $b$ ) have nothing in common with the theoretical spectra in Fig. 5. First of all, it is the maximum at energy of the trion line that persists with all spectra.

The second difference is the shape of the luminescence band (curves $a$ and $b$ ). Both the high-energy and low-energy wings of curves $a$ and $b$ demonstrate an exponential-like decrease. The broadening of the zero-plasmon band (full width at half maximum) changing from sample to sample consists of about 1-2 meV, which can be explained as a result of the spatial 


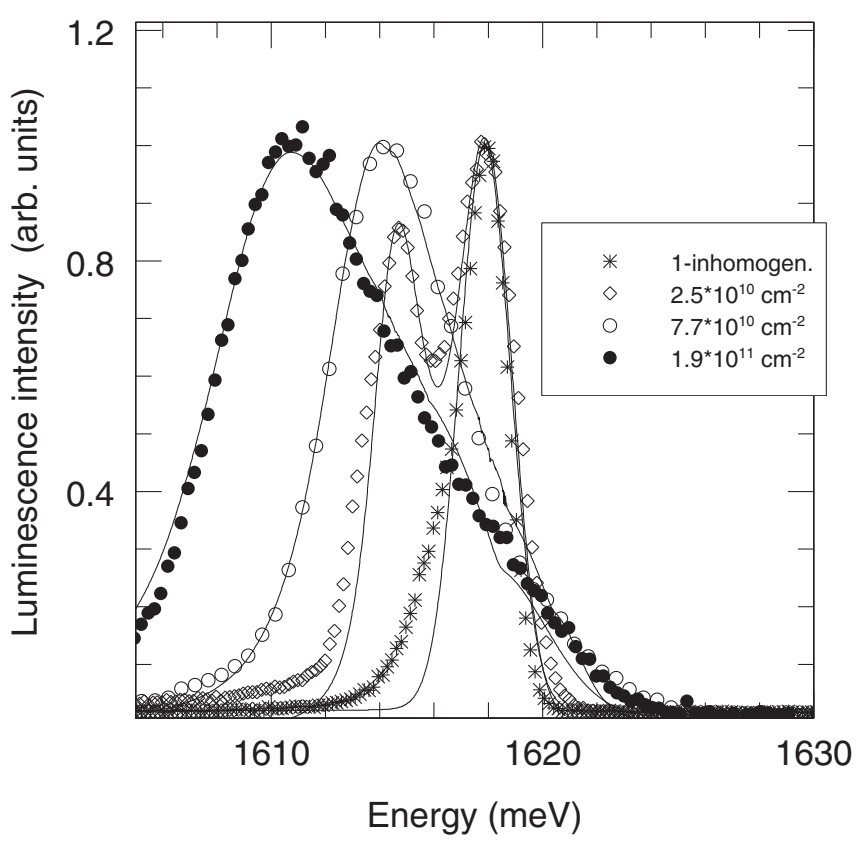

FIG. 8. Luminescence spectra taken from CdTe/CdMgTe quantum wells with different electron concentrations. Symbols are the experimental data. Curve 1 is approximated by the inhomogeneously broadened zero-plasmon band of Eq. (23). The other curves (solid lines) are approached by linear combinations of the inhomogeneously broadened zero-plasmon band given by Eq. (23) and by Eq. (24) for different electron densities. The trion spectral position $E_{\mathrm{tr}}$ is averaged over the distribution Eq. (25) with different values of $\gamma_{\text {inh }}$ above and below $E_{\mathrm{tr}}^{0}$. The $\mathrm{CdTe} / \mathrm{CdMgTe}$ quantum-well parameters given in Table I are used in calculations. The quantum-well thickness is equal to $8 \mathrm{~nm}$.

inhomogeneity of the quantum well. Curves 1 and 2 fitting curves $a$ and $b$ were obtained from Eq. (23) by averaging the trion spectral position $E_{\text {tr }}$ over the distribution

$$
\cosh ^{-2}\left[\left(E_{\mathrm{tr}}^{0}-E_{\mathrm{tr}}\right) / \gamma_{\mathrm{inh}}\right]
$$

with different values of $\gamma_{\text {inh }}$ above and below $E_{\mathrm{tr}}^{0}$, which is the trion band position in the limit of zero electron concentration. However, there exists a notable deviation of the experimental data of the curve $b$ from the simple exponential behavior in the region's low-energy tail. This deviation can be understood as the influence of free carriers, which were not taken into account in the calculations of curve 2 .

The deviation from the exponential behavior demonstrated by curve $b$ increases strongly for curves $c$ and $d$. Curves $c$ and $d$ show the strong shift of the maxima, as was shown by the theoretical curves in Fig. 5. The decreased features at the zero-plasmon trion line position show that the emission in this region of the spectra occurs only from a minor part of the quantum well.

Therefore, the observed transformation of the spectra can be considered as a result of the selective filling of the quantum well by free carriers due to their spatial inhomogeneity. We can conclude that the full trion-plasmon band is, in general, a sum of two contributions: the zero-plasmon line given by
Eq. (23) averaged over inhomogeneous distribution of the trion spectral position $E_{\text {tr }}$ of Eq. (25), and the trion-plasmon band given by Eq. (24). Although both constituents are assumed to be inhomogeneously broadened, the effect can be clearly observed only for the zero-plasmon line.

Detailed comparisons of the experimental calculated photoluminescence spectra in the energy region of the trion emission band are presented in Figs. 7 and 8. An acceptable agreement between experimental data and calculations has been obtained by fitting the values of the free-electron density and relative contribution of the quantum-well regions with and without free-electron doping.

As is seen in Figs. 7 and 8, the energy position of the broadened zero trion line does not depend on the electron concentration and coincides well with the trion PL line at low electron density.

\section{Discussion}

To describe the experimental PL spectra, we have assumed the following succession of processes: The trion arises in the spectrum of interband excitation because an exciton forms the potential well, which is able to catch an electron splitting up the lowest conduction-band level. The formation of the bound state lowers the energy of the system exciton plus the electron for the value of the binding energy, which is mainly due to exchange electron-electron interaction. The annihilation of the exciton from the bound state leads to the disappearance of the potential well. As a consequence, the electron level returns to its position at the bottom of the conduction band. The energy of the emitted photon is less than the exciton energy for the trion binding energy, which is spent for the return of the electron to the initial state.

The considered succession enables us to explain the coincidence of the energy of the trion lines in absorption and photoluminescence spectra at low electron concentration. It is also possible to assume that the formation of the charged trion leads to repulsion of the electrons of the Fermi sea, thus preventing the bound exciton from screening and stabilizing the trion state. The exchange electron-electron interaction, which is responsible for the bound state formation, cannot be screened by free carriers.

The situation with regard to the trion PL spectra looks very similar to that of the free-exciton PL spectra in $3 \mathrm{D}$ ionic crystals where the zero-phonon line is strongly suppressed. The only difference consists in the fact that, in the latter case, the role of the fastest process is played by the excitonLO-phonon interaction. Namely, this process suppresses the zero-LO-phonon exciton photoluminescence and leads to the dominant role of the LO-phonon-exciton bands in exciton luminescence.

\section{CONCLUSION}

The broadening of the trion photoluminescence bands in $\mathrm{CdTe} / \mathrm{CdMgTe}$ quantum wells as a function of the free-carrier concentration has been investigated. It has been shown that for the charged trion, the Coulomb interaction with electrons leads to the formation of a strong trion-plasmon band accompanying the trion emission line from the quantum-well regions with 
small free-electron density. In the cases of high doping, the trion-plasmon band plays the leading role in defining the trion luminescence. These spectra coincide well with the results of calculations.

\section{ACKNOWLEDGMENTS}

This work was supported in part by grants of the RFBR, the Presidium RAS, and the CNRS. *albert.klochikhin@mail.ioffe.ru

${ }^{1}$ I. Bar-Joseph, Semicond. Sci. Technol. 20, R29 (2005).

${ }^{2}$ R. B. Miller, T. Baron, R. T. Cox, and K. Saminadayar, J. Cryst. Growth 184/185, 822 (1998).

${ }^{3}$ R. T. Cox, R. B. Miller, K. Saminadayar, and T. Baron, Phys. Rev. B 69, 235303 (2004).

${ }^{4}$ B. Stebe, E. Feddi, A. Ainane, and F. Dujardin, Phys. Rev. B 58, 9926 (1998).

${ }^{5}$ F. X. Bronold, Phys. Rev. B 61, 12620 (2000).

${ }^{6}$ R. A. Suris, V. P. Kochereshko, G. V. Astakhov, D. R. Yakovlev, W. Ossau, J. Nurnberger, W. Faschinger, G. Landwehr, T. Wojtowicz, G. Karczewski, and J. Kossut, Phys. Status Solidi B 227, 343 (2001).

${ }^{7}$ A. Esser, R. Zimmermann, and E. Runge, Phys. Status Solidi B 227, 317 (2001).

${ }^{8}$ D. R. Yakovlev, V. P. Kochereshko, R. A. Suris, H. Schenk, W. Ossau, A. Waag, G. Landwehr, P. C. M. Christianen, and J. C. Maan, Phys. Rev. Lett. 79, 3974 (1997).

${ }^{9}$ V. P. Kochereschko, D. R. Yakovlev, A. V. Platonov, W. Ossau, A. Waag, G. Landwehr, and R. T. Cox, in Proceedings of ICPS-23, Berlin July 2 1-26, edited by M.Scheffler and R.Zimmerman (World Scientific, Singapore, 1996), p. 1943.
${ }^{10}$ D. Andronikov, V. Kochereshko, A. Platonov, T. Barrick, S. A. Crooker, and G. Karczewski, Phys. Rev. B 72, 165339 (2005).

${ }^{11}$ V. V. Solovyev and I. V. Kukushkin, Phys. Rev. B 79, 233306 (2009).

${ }^{12}$ R. A. Sergeev and R. A. Suris, Phys. Solid State 43, 746 (2001).

${ }^{13}$ J. H. Davies, The Physics of Low-dimensional Semiconductors: An Introduction (Cambridge University Press, Cambridge, UK, 1998).

${ }^{14}$ F. Stern, Phys. Rev. Lett. 18, 546 (1967).

${ }^{15}$ T. Ando, A. B. Fowler, and F. Stern, Rev. Mod. Phys. 54, 437 (1982).

${ }^{16}$ J. K. Jain and S. Das Sarma, Phys. Rev. B 36, 5949 (1987).

${ }^{17}$ E. H. Hwang and S. Das Sarma, Phys. Rev. B 75, 205418 (2007).

${ }^{18}$ A. Bergara, I. Nagy, and P. M. Echenique, Phys. Rev. B 55, 12864 (1997).

${ }^{19}$ S. J. Allen Jr., D. C. Tsui, and R. A. Logan, Phys. Rev. Lett. 38, 980 (1977).

${ }^{20}$ H. Al Jawhari, A. G. Kozorezov, M. Sahraoui-Tahar, J. K. Wigmore, and C. D. W. Wilkinson, Physica B 263-264, 211 (1999).

${ }^{21}$ T. Wojtowicz, M. Kutrowski, G. Karczewski, G. Cywinski, M. Surma, J. Kossut, D. R. Yakovlev, W. Ossau, G. Landwehr, and V. Kochereshko, Acta Phys. Pol. A 92, 1075 (1997). 